Journal of Computer Science 6 (1): 92-100, 2010

ISSN 1549-3636

(C) 2010 Science Publications

\title{
Land Cover Change Detection Using Texture Analysis
}

\author{
${ }^{1}$ Xiaofeng Wu, ${ }^{2}$ Fen Yang and ${ }^{2}$ Roly Lishman \\ ${ }^{1}$ Department of Aeronautical Engineering, University of Sydney, NSW 2006, Australia \\ ${ }^{2}$ Department of Computing Science, University of Aberdeen, Aberdeen, AB24 3UE, UK
}

\begin{abstract}
Problem statement: It is an important task to detect land cover changes from remotely sensed data for environmental monitoring. Although there are some applications of visual textures to the land use, they are limited to a few land cover categories with the application of one texture measure. Since land cover types are complex and often the integration of various objects, applying one texture measure to characterize land cover types is not possible. Approach: This study presented two types of texture measures for land cover types and applies them to detect possible land cover changes by discriminant analysis. The texture information of land cover types were modeled by different texture extraction methods, Laws Masks and Gabor filters. Laws Masks were designed to characterize the features in local neighborhoods. Moreover information in multi-channel of the spatial frequency domain was modeled by the Gabor filters with different orientations and spatial periods. The performance of these texture measures to detect land cover changes were evaluated by the discriminant analysis. Based on the transition matrix of land cover, the detection of land cover changes becomes to separate the land cover pair which is possible to derive conversion between them. The discriminant analysis was designed on a statistical test, which determines the contribution of the features attending the discrimination. Results: The experiments showed that this approach is capable of detecting changes and different measures are suitable to detect different changes. Conclusion: The experiment presented a textural guide for the change detection.
\end{abstract}

Key words: Discriminant analysis, land cover change, remote sensing, texture analysis

\section{INTRODUCTION}

Environmental satellite missions are designed to support a broad range of environmental monitoring applications including volcanic eruption monitoring, forest fire detection, global vegetation analysis and many other applications. The ability to detect temporal changes in images is one of the most important functions in intelligent image processing systems using remotely sensed data from the environmental satellites.

In the land use/cover change detection community, commonly used methods like image difference, changed vector analysis are not perfect. They often neglect spatial similarities of land covers and pay little attention to the neighborhood of analyzed pixels in images. Landscape images usually cover natural and semi-natural environments. The assemblages of sorts of land cover make it difficult to monitor the real changes of land covers. To deal with such complex images, where there is an excess of boundary pixels among different land covers or where there is substantial spectral overlap between different categories (Filho et al., 1996), it is better to seek a method capable of utilizing spatial and spectral information simultaneously. Texture measures are such kind of information and have achieved many applications in medical images (Sutton and Hall, 1972; Harms et al., 1986; Chen et al., 1989), document analysis (Wang and Srihari, 1989; Fletcher and Kasturi, 1988), remotely sensed image (Kurosu et al., 1999; Fukuda and Hirisawa, 1999; Angelo and Haertel, 2003) and other areas.

Since texture measures can identify the spatial organization of land covers and provide information about landscape homogeneity or heterogeneity, there are applications on segmentation and classification for textural analysis. However, most of them are limited to employing one kind of textural measures to a few land cover categories. In actual applications, textural measures attempt to quantify the spatial and spectral relationships among neighboring pixels from different angles. The extraction of textural measures is actually a mathematical scheme which converts the surface

Corresponding Author: Xiaofeng Wu, School of Aerospace, Mechanical and Mechatronic Engineering, University of Sydney, NSW 2006, Australia Tel: +61 290367053 
roughness of land cover categories into numerical values. It is the mathematical converting way that determines textural information captured.

Therefore many algorithms are developed to capture textural information from different angles. Although there are many textural measures available, their classification effects to different land cover categories are different. It is not enough to use only one kind of textural measures to semi-natural environment. Different textural measures can capture different variations of land cover types. Some measures may be good at capture local high variance like in a forested environment, while others are able to capture directional features. The variety of local features in the landscape image makes the single textural measure lose some information. Applying more than one textural measure can make up a loss of each other. Therefore applying more than one measure can achieve better quality for distinguishing the land cover categories. In this study, we will discuss textural measures from the angles of the spatial and the spatial frequency domains.

Specifically designed spatial filters may capture some special details of certain land cover types. This facility could be used to enhance wanted details or suppress unwanted details. In the applications of monitoring land cover, spatial filters are mostly designed to remove noise resulting from the spectral signature or separate mixed pixels such as clouds (Giri et al., 2003) from other land cover types or mask one kind of land cover from the others (Morisette et al., 1996). In this study specially designed filters-Laws Masks, are used to capture the characteristics of certain land cover types so as to distinguish different types of land cover.

In addition, Gabor filters are well recognized in the recent past as a joint spatial and spatial frequency representation of textures. Daugman (1980) has shown that Gabor filters have optimal joint localization in both the spatial and the spatial frequency domains. Moreover they are analogous to the textural visual perception. Gabor decompositions have been proposed as an analysis tool for texture (Bigun and du Buf, 1994) which is inspired by the psychological research of the human visual system (Malik and Perona, 1990). Gabor filters are employed in many researches, such as texture segmentation (Tsai et al., 2001; Weldon et al., 1996), texture classification (Manjunath and Ma, 1996) and texture extraction (Kruizinga and Petkov, 1999; Koperski et al., 2002). Koperski et al. (2002); Shapiro and Stockman (1991) applies Gabor wavelets in a system for data mining of remotely sensed images. Manjunath and Ma (1996) used Gabor filter-based multi-resolution representations to extract texture information. While many researches pay attention to the role of Gabor filters in texture processing, most of them are limited to artificial features or textures in standard texture libraries. There are few applications of Gabor filters to the natural landscape images. Based on the property of Gabor filters, which are able to extract information from the spatial and spatial frequency domain concurrently, the performance of Gabor filters in the characterization of land cover types is discussed in this study as well.

The applicability of different textural measures on the discrimination of land cover categories is evaluated by the discriminant analysis. Land cover change is affected by natural and human activities. It is difficult to track human interventions, so when we discuss environmental changes in this study, we mean the natural and ecological changes, which happen to the land cover types. The problem of change detection can be transferred to the classification of the land cover types. For example, an "arable" land is possible to transfer to the type of "good rough grassland" partially or completely under natural ecological trends without human management. In this case, if the texture measures of this new polygon are classified to the category of "good rough grassland" by the discriminant analysis, then the change happened to the original polygon is tracked.

\section{MATERIALS AND METHODS}

Laws masks: A common approach to generating texture feature is to use local masks to capture local information in images. Laws developed a texture energy approach that measures the amount of variation within a fixed-size window. A set of $5 \times 5$ convolution masks are defined to compute texture energy, which is then represented by a vector for each pixel of the image being analyzed. These vectors are the convolution results of masks and the image. The masks are achieved through the following vectors:

$$
\begin{aligned}
& \text { L5 (Level) }=\left|\begin{array}{lllll}
1 & 4 & 6 & 4 & 1
\end{array}\right| \\
& \text { E5 (Edge) }=\left|\begin{array}{lllll}
-1 & -2 & 0 & 2 & 1
\end{array}\right| \\
& \text { S5 (Spot) }=\left|\begin{array}{llllr}
-1 & 0 & 2 & 0 & -1
\end{array}\right| \\
& \text { R5 (Ripple) }=\left|\begin{array}{lllll}
1 & -4 & 6 & -4 & 1
\end{array}\right|
\end{aligned}
$$

These vectors imitate four different spatial distributions. And like the names meaning, the L5 vector gives a centre-weighted local average. The E5 vector detects edges, the S5 vector detects spots and the $\mathrm{R} 5$ vector detects ripples. The $2 \mathrm{D}$ convolution masks are obtained by computing outer products of pairs of 
vectors. For example, the mask L5E5 is computed as the product of L5 and E5 as:

$$
\left[\begin{array}{l}
1 \\
4 \\
6 \\
4 \\
1
\end{array}\right] \times\left[\begin{array}{lllll}
1 & -4 & 6 & -4 & 1
\end{array}\right]=\left[\begin{array}{ccccc}
1 & -4 & 6 & -4 & 1 \\
4 & -16 & 24 & -16 & 4 \\
6 & -24 & 36 & -24 & -6 \\
4 & -16 & 24 & -16 & 4 \\
1 & -4 & 6 & -4 & 1
\end{array}\right]
$$

These masks can measure different contents of the images. For example, E5L5 measures horizontal edge content and L5E5 measures vertical edge content. The average of these two measures is the total edge content. Because some masks are symmetric, they are combined and replaced by the average. Finally only nine masks are used to extract texture measures. They are:

\section{L5E5/ E5L5 L5S5/S5L5 \\ L5R5/R5L5 E5E5 \\ E5S5/S5E5 E5R5/R5E5 \\ S5S5 S5R5/R5S5 \\ R5R5}

These masks are subsequently convolved with the image to highlight its microstructure which generates the energy map. Nine energy maps are obtained for every image. Let $F_{k}[i, j]$ be the result of filtering with the kth mask at pixel $[i, j]$. Then the texture energy map $\mathrm{E}_{\mathrm{k}}$ for filter $\mathrm{k}$ is defined by:

$E_{k}[r, c]=\sum_{j=c-7}^{c+7} \sum_{i=r-7}^{r+7}\left|F_{k}[i, j]\right|$

where, $(r, c)$ is the centre point of the filtering window. The size of the window depends on the class of imagery. A $15 \times 15$ window is used for natural scenes (Comber et al., 2001). Each texture energy map is a full image, representing the application of the kth mask to the input image. Statistical measures, mean, standard deviation, skewness, kurtosis and energy, are regarded as texture measures of the energy maps and form a vector for further classification.

Gabor filters: Gabor filters are a kind of operators that can capture the local information in an image optimally. And research show that Gabor filters mimic the biological perception of texture and share many properties with the human visual system (Raghunathan and Acton, 2000). This property of Gabor filters makes them capable of reaching the minimum bound for simultaneous localization in the spatial and spatial/frequency domains (Tsai et al., 2001). Moreover Gabor filters bank composing of a set of kernels can provide a complete cover of spatial/frequency domain so that it can generate a versatile model for texture description of various land cover. Because Gabor filters can be calculated at different spatial frequencies, it can capture similarities in images of different scales.

Biological research shows simple cells in the primary visual cortex of primates play an important role in the perception of the texture. The Gabor filters perform the same role with the simple cells when perceiving textures. The texture perceived by people is actually the response of the correlation of Gabor filters with the image. Therefore, choosing suitable parameters for Gabor filters can extract specified textures.

A Gabor filter is a complex sinusoid modulated by a Gaussian envelope. Its general one-dimensional form in Cartesian co-ordinate is:

$g(x)=\frac{1}{\sqrt{2 \pi \sigma}} \cdot \exp \left(\frac{-x^{2}}{2 \sigma^{2}}\right) \cdot \exp \left(i 2 \pi \frac{x}{\lambda}\right)$

Where:

$\sigma=$ The standard deviation of the Gaussian function, determines the size of the receptive field

$\lambda=$ The wavelength of the complex sinusoid

$2 \pi / \lambda$ determines the preferred spatial frequency of the receptive field function.

The 1-D Gabor function of Cartesian co-ordinate in the spatial frequency domain is as follows:

$\mathrm{G}(\mathrm{u})=\exp \left[\frac{-\sigma^{2}\left(\mathrm{u}-\mathrm{u}_{\mathrm{c}}\right)^{2}}{2}\right]$

Where:

$\mathrm{u}=$ The spatial frequency

$\mathrm{u}_{\mathrm{c}}=$ The preferred spatial frequency with value $2 \pi / \lambda$ as defined above

The 1-D Gabor function may be extended to 2-D as follows:

$g(x, y)=\frac{1}{2 \pi \sigma^{2}} \cdot \exp \left[-\frac{1}{2}\left(\frac{x^{2}+y^{2}}{\sigma^{2}}\right)\right] \cdot \exp \left[i 2 \pi\left(\frac{x+y}{\lambda}\right)\right]$

where, $\sigma$ and $\lambda$ are defined as above. Its spatial frequency domain in Cartesian co-ordinate is given by:

$G(u, v)=\exp \left[\frac{-\sigma^{2}\left(u-u_{c}\right)^{2}}{2}\right] \cdot \exp \left[\frac{-\sigma^{2}\left(v-v_{c}\right)^{2}}{2}\right]$ 
Where:

$\mathrm{u}$ and $v=$ Spatial frequencies along the $\mathrm{x}$ and $\mathrm{y}$ axis

$\mathrm{u}_{\mathrm{c}}$ and $v_{\mathrm{c}}=$ The selected spatial frequencies along the $\mathrm{x}$ and y directions respectively

Equation 7 could be denoted in polar co-ordinate as well. There are many advantages in polar form over the standard form. Haley and Manjunath (1991) pointed that compared with the standard form the Gabor filter in polar form has a narrower response at low frequencies and a wider response at high frequencies. This means the range of parameters in polar co-ordinate can make a more uniform coverage of the frequency domain with less overlap at low frequencies and smaller gaps at high frequencies. Also the polar form is more suitable for rotation invariant analysis which is a requirement to describe natural objects in landscape images. Another advantage of the polar form is that the parameters of the Gabor filter are more easily determined than the standard form. The Gabor filter in polar form will be applied in this study.

MPEG-7 homogeneous texture descriptor: MPEG-7 is a standard for describing the multimedia content data that supports some degree of interpretation of the information's meaning, which can be passed onto, or accessed by, a device or a computer code. The application fields covered include geographic information systems, remote sensing, multimedia editing and so on. It describes three texture descriptors, a Homogeneous Texture Descriptor (HTD), an Edge Histogram Descriptor (EHD) and a Perceptual texture Browsing Descriptor (PBD).

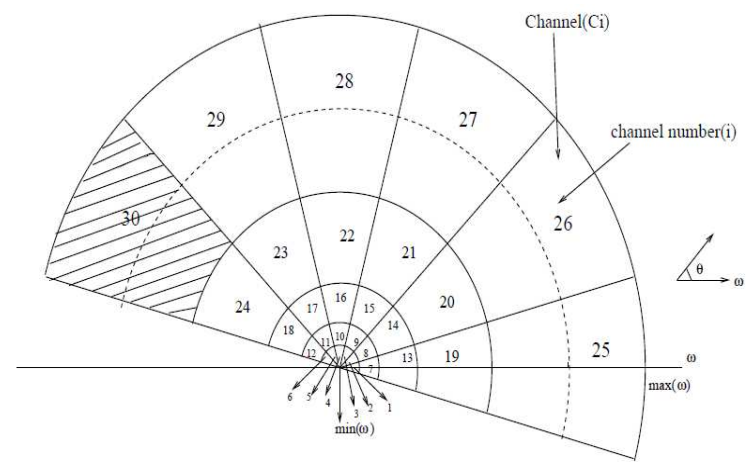

Fig. 1: Frequency partition of the Gabor filter bank with ID's of the feature channels $C$ in the spatial frequency domain. (f)max and $\min (\mathrm{f})$ denote the maximum and minimum frequency in the spatial frequency domain respectively
The HTD provides a precise quantitative representation of texture that is useful for similarity analysis. The descriptor is derived from filtering with original image using scale and orientation selective kernels which create a filter bank of Gabor functions. Gabor functions in the spatial frequency domain in polar form make them convenient to generate the parameters of Gabor kernels. The Spatial frequency domain in polar co-ordinate could be partitioned into 30 channels with equal divisions in the angular direction (at $30^{\circ}$ intervals) and octave division in the radial direction (5 octaves) as shown in Fig. 1. The channel index $i$ can be denoted as $i=6^{*} s+r+1$. Here $s$ is the radial index with $s \in\{0,1,2,3,4\}$ and $r$ is the angular index with $r \in\{0,1,2,3,4,5\}$.

Every feature channel in the spatial frequency domain is modeled using a 2-D Gabor function of polar form as follows:

$\mathrm{G}_{\mathrm{P}_{\mathrm{s}, \mathrm{r}}}(\mathrm{f}, \theta)=\exp \left[\frac{-\left(\mathrm{f}-\mathrm{f}_{\mathrm{s}}\right)^{2}}{2 \mathrm{~S}_{\mathrm{f}_{\mathrm{s}}}^{2}}\right] \cdot \exp \left[\frac{-\left(\theta-\theta_{\mathrm{r}}\right)^{2}}{2 \mathrm{~S}_{\theta_{\mathrm{r}}}^{2}}\right]$

Where:

$\mathrm{f}=$ the frequency in radial direction

$\theta=$ the angular direction

Centre frequency of octave bandwidth:

$\mathrm{f}_{\mathrm{s}}=\frac{3}{4} \cdot(\max (\mathrm{f})-\min (\mathrm{f})) \cdot 2^{-\mathrm{s}}$

where radial index $s \in\{0,1,2,3,4\} . \max (f)$ is the maximum frequency of the image and has wavelength 2 pixels/cycle. min(f) is the minimum frequency of the image and has wavelength 1 picture/cycle. Angular direction $\theta_{\mathrm{r}}=30^{\circ} * \mathrm{r}$, where angular index $r \in\{0,1,2,3,4,5\} . \quad S_{f_{s}}$ and $S_{\theta_{r}}$ are the standard deviations of the Gabor function in the radial direction and the angular direction respectively. In the angular direction, $S_{\theta_{r}}$ has a constant value as follows:

$\mathrm{S}_{\theta_{\mathrm{r}}}=\frac{15^{\circ}}{\sqrt{2 \ln 2}}$

In the radial direction, $\mathrm{S}_{\mathrm{f}_{\mathrm{s}}}$ depends on the octave bandwidth as follows:

$\mathrm{S}_{\mathrm{s}_{\mathrm{r}}}=\frac{\mathrm{B}_{\mathrm{s}}}{2 \sqrt{2 \ln 2}}$ 
where, $B_{s}$ is the octave bandwidth whose value is $(\max (\mathrm{f})-\min (\mathrm{f})) \cdot 2^{-(\mathrm{s}+1)}$. The HTD generates a feature vector $T D$ constituted by the mean value $f_{D C}$ and standard deviation $\mathrm{f}_{\mathrm{SD}}$ of the original image as well as the energies $e_{i}$ and their standard deviations $d_{i}$ of the Gabor filtered images:

$\mathrm{TD}=\left[\mathrm{f}_{\mathrm{DC}}, \mathrm{f}_{\mathrm{SD}}, \mathrm{e}_{1}, \mathrm{e}_{2}, \ldots, \mathrm{e}_{30}, \mathrm{~d}_{1}, \mathrm{~d}_{2}, \ldots \mathrm{d}_{30}\right]$

Discriminant analysis: Discriminant Analysis (DA) is a statistical technique that makes use of the Analysis Of Variance (ANOVA) to determine whether a set of variables can separate different groups. The difference is determined with regard to the means of the variables. DA is used in the texture distinguishing process.

The purpose of DA is to generate a discriminant function or a set of discriminant functions if there are more than two groups. The functions are based on linear combinations of the independent variables and provide the best discrimination between the groups. The discriminant analysis function determines whether groups differ with regard to the means of independent variables. The functions are generated from a sample of cases for which group membership is known and meet the requirement that the ratio $\mathrm{SS}_{\text {between }} / \mathrm{SS}_{\text {total }}$ is as large as possible when carrying out the one-way ANOVA. $\mathrm{SS}_{\text {total }}$ is the total sum of squares, which is a measure of the total dispersion of the scores around the grand mean and can be divided into two components:

$\mathrm{SS}_{\text {total }}=\mathrm{SS}_{\text {between }}+\mathrm{SS}_{\text {within }}$

Where:

$\mathrm{SS}_{\text {between }}=$ The dispersion of the group means around the grand mean

$\mathrm{SS}_{\mathrm{within}}=$ The dispersion around the group mean

If there are $\mathrm{m}$ groups and $\mathrm{n}$ independent variables, the discriminant functions could be described as:

$\mathrm{Y}=\beta \mathrm{X}+\varepsilon$

Where:

$\mathrm{Y}=$ Denotes the group member vector

$\beta=$ Denotes the discriminant coefficients vector, which maximizes the distance between the means of the dependent variables. In fact discriminant coefficients act as weights of features in discriminating different groups

$\mathrm{X}=$ Denotes the independent variables

$\varepsilon=$ Denotes error vector

Their forms are as follows:

$\mathrm{Y}=\left(\mathrm{y}_{1}, \mathrm{y}_{2}, \ldots, \mathrm{y}_{\mathrm{m}}\right)$

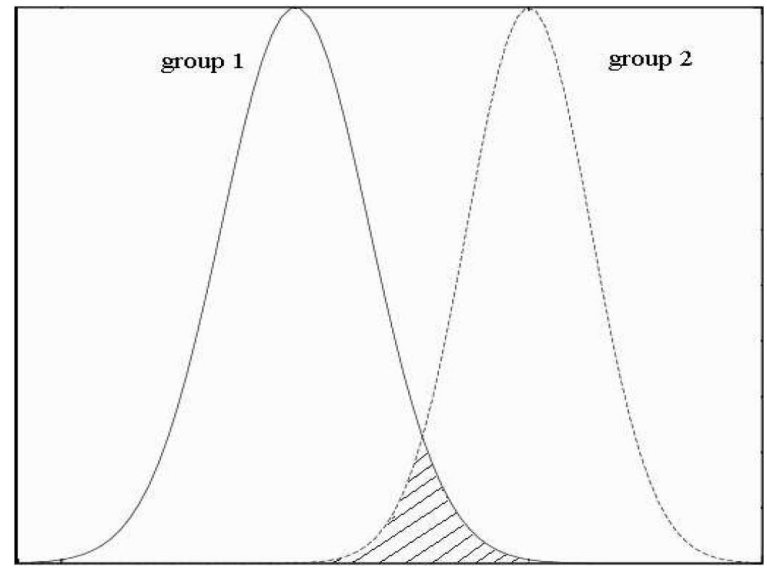

Fig. 2: The distributions of two groups

$\mathrm{X}=\left(\mathrm{x}_{1}, \mathrm{x}_{2}, \ldots, \mathrm{x}_{\mathrm{n}}\right)$

$\beta=\left(\begin{array}{ccccc}\beta_{10} & \beta_{11} & \beta_{12} & \cdots & \beta_{1 \mathrm{n}} \\ \beta_{20} & \beta_{21} & \beta_{22} & \cdots & \beta_{2 \mathrm{n}} \\ \vdots & \vdots & \vdots & \vdots & \vdots \\ \beta_{\mathrm{m} 0} & \beta_{\mathrm{m} 1} & \beta_{\mathrm{m} 2} & \cdots & \beta_{\mathrm{mn}}\end{array}\right)$

$\varepsilon=\left(\varepsilon_{1}, \varepsilon_{2}, \ldots \varepsilon_{\mathrm{m}}\right)$

For each of the categories in DA, there will be a distribution, such as a normal distribution, for the members of that category. The distributions of different groups usually overlap like the Fig. 2 showing that there is an overlap (shade part) between the two groups. The goal of the DA is to find values for the discriminant coefficients and weigh the independent variables such that the overlaps among distributions of groups are minimized.

The values of independent variables in the known cases are important to the DA. Their contribution to the discrimination is weighed as the discriminant coefficients. A statistical test like f-test assesses whether the independent variables reliably discriminate the categories of the dependent variables.

F-value is a measure of the extent to which a variable makes a unique contribution to the discrimination. It is computed as the ratio of the between-groups variance over the pooled within group variance:

$$
\mathrm{F}_{\text {value }}=\frac{\mathrm{SS}_{\text {between }}}{\mathrm{SS}_{\text {within }}}
$$


However in ANOVA, this is expressed in a statistic known as Wilk's lambda $(\Lambda)$. It is expressed as follows:

$$
\Lambda=\frac{\mathrm{SS}_{\text {between }}}{\mathrm{SS}_{\text {total }}}
$$

A relatively small $\Lambda$ implies a relatively large dispersion among the group means. Thus smaller values of $\Lambda$ are more likely to be significant. During the analysis of DA, only those independent variables which make the $\Lambda$ small enough are able to take part in the analysis. In other words Wilk's Lambda determines the reliability of independent variables. The more reliable the variable, the more it affects the DA.

The quantities of the contribution for independent variables are measured as the discriminant coefficients. Generally the discriminant coefficients are determined by the least squares estimates. It attempts to minimize the error vectors in the Eq. 14 and uses the following formula:

$$
\mathrm{Q}(\beta)=\sum_{\mathrm{i}=1}^{\mathrm{m}} \varepsilon_{\mathrm{i}}^{2}=\sum_{\mathrm{i}=1}^{\mathrm{m}}\left(\mathrm{Y}_{\mathrm{i}}-\beta \mathrm{x}_{\mathrm{i}}\right)^{2}
$$

where, $Q(\beta)$ is the residual sum of squares which measures the remaining/residual variation of data.

The coefficient matrix $\beta$ obtained is labeled as $\hat{\beta}$ which minimizes $\mathrm{Q}(\beta)$. Thus, the discriminant functions are fitted as:

$$
\hat{y}_{i}=x_{i}^{\prime} \hat{\beta}=\hat{\beta}_{0}+\hat{\beta}_{1} x_{i 1}+\ldots \hat{\beta}_{p} x_{i n} \quad i=1,2, \ldots, m
$$

\section{RESULTS}

Ten kinds of land cover types, which are shown in Table 1, have been examined, as these land cover types are ecologically "similar". From the Table 1 it is clear that under every type, there are more detailed subclasses. Although according to Comber ${ }^{[23]}$ these ten types of land cover are transferable, these versatile appearances of the subclasses make the classification more difficult. The possible transitional matrix of these land cover types is listed in Table 2. Under this guidance, detecting changes of some land covers becomes to distinguish the possible land covers from original land cover. In this experiment training and testing data are used half and half to avoid bias.

Experimental data: The data in this experiment is from remotely sensed images covering the Elgin area in northeast Scotland. The image has been manually segmented into different land cover types by expert interpreters who gave every pixel in the image a land code representing the land cover type of the pixel. Images only containing single land type are required so that different information from different land cover types could be compared and analyzed without the influence of other land cover types. According to the given code polygons are obtained by using the flood fill method.

Table 1: Land-cover types description

\begin{tabular}{lcl}
\hline Land cover category & Land over code & Main feature \\
\hline Arabic field & 10 & Arabic field (no rock, no farms, no trees) \\
Good rough grassland & 150 & Smooth grass/rushes (no rock, no tress) \\
& 151 & Smooth grass/rushes (no rock, tress) \\
& 155 & Smooth grass/low scrub (no tock, no trees) \\
& 156 & Smooth grass/low scrub (no tock, trees) \\
Heather moorland & 160 & Undifferentiated smooth grass (no rock, no tress) \\
& 161 & Undifferentiated smooth grass (no rock, tress) \\
& 110 & Dry heather moor (no rock, no burring, no tress) \\
& 11 & Dry heather moor (no rock, no burring, tress) \\
Poor rough grassland & 112 & Dry heather moor (no rock, burring, no trees) \\
Bracken & 120 & Wet heather moor (no rock, no burning, no tress) \\
Mixed woodland & 130 & Undifferentiated heather moor (no rock, no burring, no trees) \\
Scrub & 131 & Undifferentiated heather moor (no rock, no burring, trees) \\
Peatland & 132 & Undifferentiated heather moor (no rock, burring, no trees) \\
& 140 & Undifferentiated Nardus/Molinia (no rock, no tress) \\
& 170 & Undifferentiated bracjen (no rock, no trees) \\
Semi-natural coniferous & 79 & Undifferentiated mixed woodland (trees) \\
Broadleaved & 82 & Undifferentiated low scrub \\
& 180 & Blanket bog/peatland veg. (erosion, no trees) \\
& 182 & Blanket bog/peatland veg. (no erosion, no trees) \\
& 183 & Blanket bog/peatland veg. (no erosion, trees) \\
& 186 & Blanket bog/peatland veg. (dubh lochans)
\end{tabular}


Table 2: A transition matrix of natural ecological trends of land cover

\begin{tabular}{|c|c|c|c|c|c|c|c|c|c|c|c|c|c|}
\hline $\begin{array}{l}0=\text { unlikely } \\
1=\text { possible } \\
2=\text { likely } \\
\text { (From) }\end{array}$ & Main features & Codes & To & $\begin{array}{l}\text { Arable } \\
1\end{array}$ & $\begin{array}{l}\text { Good } \\
\text { rough } \\
\text { grassland } \\
2\end{array}$ & $\begin{array}{l}\text { Heather } \\
\text { moorland } \\
3\end{array}$ & $\begin{array}{l}\text { Poor } \\
\text { rough } \\
\text { grassland } \\
4\end{array}$ & $\begin{array}{l}\text { Bracken } \\
5\end{array}$ & $\begin{array}{l}\text { Mixed } \\
\text { woodland } \\
6\end{array}$ & $\begin{array}{l}\text { Scrub } \\
7\end{array}$ & $\begin{array}{l}\text { Peatland } \\
8\end{array}$ & $\begin{array}{l}\text { Semi- } \\
\text { natural } \\
\text { coniferous } \\
9\end{array}$ & $\begin{array}{l}\text { Broadleaved } \\
10\end{array}$ \\
\hline $\begin{array}{l}\text { Arable } \\
\text { An }\end{array}$ & Arable land & 100 & 1 & & 2 & & 1 & 1 & 1 & 1 & & & 1 \\
\hline $\begin{array}{l}\text { Good rough } \\
\text { grassland }\end{array}$ & $\begin{array}{l}\text { Smooth G (rushes, } \\
\text { crub, undiff.) }\end{array}$ & $\begin{array}{l}150,151,15,5 \\
156,160,161\end{array}$ & 2 & & & & 1 & 2 & 1 & 2 & & & 1 \\
\hline $\begin{array}{l}\text { Heather } \\
\text { moorland }\end{array}$ & HM (wet, dry undiff.) & $\begin{array}{l}110,111,112,120 \\
130,131,132\end{array}$ & 3 & & & & & 1 & 2 & 2 & 1 & 2 & 2 \\
\hline Poor rough grassland & Undiff. Coarse G & 140 & 4 & & & 1 & & 1 & & 1 & & & 1 \\
\hline Bracken & $\begin{array}{l}\text { Undiff. smooth G } \\
\text { with bracken }\end{array}$ & 170 & 5 & & & & & & 1 & 2 & & & 1 \\
\hline Mixed woodland & $\begin{array}{l}\text { Undiff. mixed } \\
\text { woodland (area) }\end{array}$ & 79 & 6 & & & & & & & 1 & & & 1 \\
\hline Scrub & Undiff. low scrub & 82 & 7 & & & & & & 1 & & & 1 & 2 \\
\hline Peatland & Blanket bog & $180,182,183,186$ & 8 & & & 1 & 1 & & & 1 & & & \\
\hline $\begin{array}{l}\text { Semi-natural } \\
\text { coniferous }\end{array}$ & $\begin{array}{l}\text { Semi-nat conif } \\
\text { woodland }\end{array}$ & 73 & 9 & & & & & & 1 & 1 & & & \\
\hline Broadleaved & Broadleaved W & 76 & 10 & & & & & & & 1 & & & \\
\hline
\end{tabular}

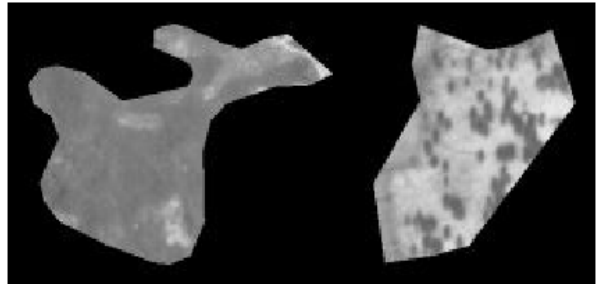

Fig. 3: Left polygon is labeled as Dry Heather Moor and right polygon is labeled as Smooth Grass/rushes

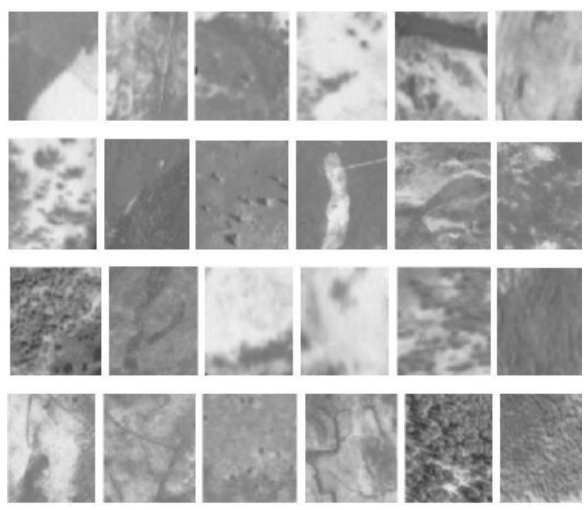

Fig. 4: An example of polygons in training data set. From left to right and up to down, the land cover types are listed in the order of Table 1

Finally the image covering Elgin is split into many polygons. Figure 3 shows two examples of polygons produced by the flood fill method. Each polygon only contains one land code, i.e., one land cover type, but there may exist many polygons associated with one land code because polygons with the same land code may be not adjacent. Thus it is ready to derive land cover texture information from these different polygons for further analysis.

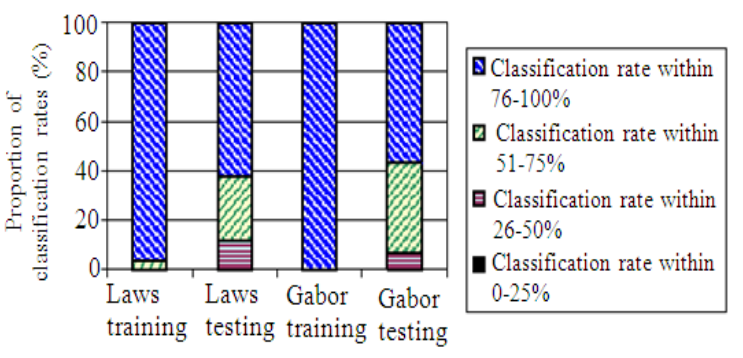

Fig. 5: Experiments results of laws masks and Gabor filters

Experiment results: There are a total of 475 polygons of 10 land types used in the experiment. Figure 4 shows an example of some textures used in the training dataset. According to the method mentioned above polygons obtained from floodfill method are correlated with 9 Laws Masks and 30 Gabor filters respectively. Texture features are derived from the filtered images and then those features are input to a linear regression discriminant classifier. The final results of these two texture measures are shown in Fig. 5.

\section{DISCUSSION}

Results show that these two kinds of texture measures are promising to detect land cover changes. Most of the correct classification rates are higher than $80 \%$. Although they are both bandpass filters, the difference is the division of the spatial frequency domain. Each filter under the same filter banks has its own spatial frequency range and therefore captures different local textural features. Laws masks are designed to extract special characteristics in the neighborhood. Gabor filters are capable of emulating human perception and capturing spatial information and spatial frequency information at the same time. 


\section{J. Computer Sci., 6 (1): 92-100, 2010}

Through the different divisions of the spatial frequency domain, three different filter banks are designed and they correspond to different spectral information. The textural information extracted by each filter for each land cover texture is described as a feature vector for classification.

Most of the land cover classifications apply only one single texture extraction scheme to obtain textural features. For a few major land cover categories, this may be enough. However for our study, a number of subtle land cover textures make this not possible to capture subtle variations. Therefore we apply two different filter banks: Laws masks and Gabor filters to capture these subtle variations as much as possible. Among the two filter banks, as far as the author is concerned, applying Gabor filters which are built from the polar form of the suggested MPEG-7 to land cover texture classification is the first time. The two filter banks characterize textures from different angles and thus are suitable to distinguish different textures.

Although it seems Gabor filters are better to tell these 10 land covers, due to the complexity of land covers, applying more than one texture measure is more reasonable. It is better to examine the abilities of different texture measures and choose the one with the best performance when dealing with complex land cover images.

\section{CONCLUSION}

The experiment shows that the textural measures are able to discriminate the land cover types although the discriminant quality could be improved further. The representations of various textural measures are different for the same land cover category because the attention of each kind of measures is different. The features of the land cover category are thus captured by different textural measures. However it is not clear which aspect reflects the most important characteristic of the category, it is wise to compare the performance of different textural measures.

The suggestion of choosing suitable textural measures for the future change detection is based on a statistical analysis. Its reliability is tested by an analysis of variance of the features. This statistical test can guarantee the features which make real contributions to the discrimination staying in the process. Moreover it can get rid of those redundant features which cannot provide further discriminant information at the same time. This significance level really makes the discriminant results robust.

In general it is a promising approach to apply textural measures for the land cover change detection. It can achieve the best discriminant result if employing the textural measure with the best discriminant analysis to the pairs between which could have "ecological" changes.

\section{REFERENCES}

Angelo, N. and V. Haertel, 2003. On the application of gabor filtering in supervised image classification. Int. J. Remote Sens., 24: 2167-2189. DOI: 10.1080/01431160210163146

Chen, C.C., J.S. Daponte and M.D. Fox, 1989. Fractal feature analysis and classification in medical imaging. IEEE Trans. Med. Imag., 8: 133-142. DOI: $10.1109 / 42.24861$

Comber, A. et al., 2001. Methodologies and Approaches for Automated Land Cover Change Detection. In: Innovations in GIS 8: Spatial Information and the Environment, Halls, P. (Ed.)., 1st Edn., Taylor and Francis, CRC Press, London, ISBN: 978-0415253628, pp: 37-51.

Bigun, J. and J. du Buf, 1994. N-folded symmetries by complex moments in Gabor space. IEEE Trans. Patt. Anal. Mach. Intel., 16: 80-87. DOI: 10.1109/34.273714

Daugman, J., 1980. Two-dimensional spectral analysis of cortical receptive field profiles. Vis. Res., 20: 847-856. DOI: 10.1016/0042-6989(80)90065-6

Filho, O., P. Treitz, E. Soulis, P. Howarth and N. Kouwen, 1996. Texture processing of synthetic aperture radar data using second-order spatial statistics. Comput. Geosci., 22: 27-34. DOI: 10.1016/00983004(95)0054-2

Fletcher, J.A. and R. Kasturi, 1988. A robust algorithm for text string separation from mixed text/graphics images. IEEE Trans. Image Anal. Mach. Intel., 10: 910-918. DOI: 10.1109/34.9112

Fukuda, S., H. Hirisawa, 1999. A wavelet-based texture feature set applied to classification of multifrequency polarimeter SAR images. IEEE Trans. Geosci. Remote Sens., 37: 2282-2286. DOI: 10.1109/36.789624

Giri, C., P. Defourny and S. Shrestha, 2003. Land cover characterization and mapping of continental southeast Asia using multi-resolution satellite sensor data. Int. J. Remote Sens., 24: 4181-4196.

Haley, G.M. and B.S. Manjunath, 1999. Rotationinvariant texture classification using a complete space-frequency model. IEEE Trans. Image Process., 8: 255-269. DOI: 10.1109/83.743859

Harms, H., U. Gunzer and H.M. Aus, 1986. Combined local color and texture analysis of stained cells. Comput. Vis. Graph. Image Process., 33: 364-376. DOI: 10.1016/0734-189X(86)90183-0 
Koperski, K., G. Marchisio, C. Tusk and S. Aksoy, 2002. Interactive models for semantic labeling of satellite images. Proceedings of SPIE Annual Meeting, Earth Observing System Session, July 711, Seattle, WA., pp: 423-434. DOI: $10.1117 / 12.452068$

Kruizinga, P. and K. Petkov, 1999. Nonlinear operator for oriented texture. IEEE Trans. Image Process., 8: 1395-1407. DOI: 10.1109/83.791965

Kurosu, T., S. Uratsuka, H. Maeno, T. Kozu, 1999. Texture statistics for classification of land use with multitemporal JERS-1 SAR single-look imagery. IEEE Trans. Geosci. Remote Sens., 37: 227-235. DOI: $10.1109 / 36.739157$

Malik, J. and P. Perona, 1990. Preattentive texture discrimination with early vision mechanisms. J. Opt. Soc. Am., 7: 923 -932. PMID: 2338600

Manjunath, B. and W. Ma, 1996. Texture features for browsing and retrieval of image data. IEEE Trans. Patt. Anal. Mach. Intel., 18: 837-842. DOI: $10.1109 / 34.531803$

Morisette, J.T., H. Chesire, C. Stallings and S. Khorram, 1996. Creating an Urban Mask Raster Image for Vector Streets Files. In: Raster imagery in Geographic Information Systems, Morain, S. and S. Lopez Baros (Eds.). High Moutain Press, Santa Fe, New Mexico, USA., ISBN: 978-1566900973, pp. 172-178.

Raghunathan, B. and S.T. Acton, 2000. Content based retrieval for remotely sensed imagery. Proceedings of the IEEE Southwest Symposium on Image Analysis and Interpretation, August 2002, Austin, USA. DOI: 10.1109/IAI.2000.839592
Shapiro, L.G. and G.C. Stockman, 1991. Computer Vision. Prentice-Hall Inc., New Jersey, ISBN: 9780130307965, pp: 608.

Sutton, R., E.L. Hall, 1972. Texture measures for automatic classification of pulmonary disease. IEEE Trans. Comput., 21: 667-676. DOI: 10.1109/T-C.1972.223572

Tsai, D., S. Wu and M. Chen, 2001. Optimal Gabor filter design for texture segmentation using stochastic optimization. Image Vis. Comput., 19: 299-316. DOI: 10.1016/S0262-8856(00)000780

Wang, D. and S.N. Srihari, 1989. Classification of newspaper image blocks using texture analysis. Comput. Vis. Graph. Image Process., 47: 327-352. DOI: 10.1016/0734-189X(89)90116-3

Weldon, T., W. Higgins and D. Dunn, 1996. Efficient Gabor filter design for texture segmentation. Patt. Recog., 29: 2005-2015. DOI: 10.1016/S00313203(96)00047-7 